\title{
A nomogram combining PPAR $\gamma$ expression profiles and clinical factors predicts survival in patients with hepatocellular carcinoma
}

\author{
XIAOLU ZHOU ${ }^{1,2}$, YAJING CHI $^{3}$, ZHIYUAN DONG ${ }^{1,2}$, TAO TAO $^{4}$, \\ XIN ZHANG $^{5}$, WENSHENG PAN ${ }^{2}$ and YEMENG WANG ${ }^{6}$
}

\begin{abstract}
${ }^{1}$ Department of Clinical Medicine, The Medical College of Qingdao University, Qingdao, Shandong 266071;
${ }^{2}$ Department of Gastroenterology, Zhejiang Provincial People's Hospital, People's Hospital of Hangzhou Medical College, Hangzhou, Zhejiang 310014; ${ }^{3}$ Department of Clinical Medicine, Shandong First Medical University and Shandong Academy of Medical Sciences, Jinan, Shandong 271016; ${ }^{4}$ Hithink Flush Information Network Co., Ltd., Hangzhou, Zhejiang 310000; ${ }^{5}$ Department of Pathology, Zhejiang Provincial People's Hospital, People's Hospital of Hangzhou Medical College, Hangzhou, Zhejiang 310014; ${ }^{6}$ Department of Hepatobiliary Surgery, Zhuji People's Hospital of Zhejiang Province, Zhuji, Zhejiang 311800, P.R. China
\end{abstract}

Received June 25, 2020; Accepted January 22, 2021

DOI: $10.3892 / 01.2021 .12581$

\begin{abstract}
Hepatocellular carcinoma (HCC) is the most common primary liver cancer with poor prognosis. Peroxisome proliferator-activated receptor $\gamma$ (PPAR $\gamma)$ is involved in the development of various tumor types. However, its role in hepatocellular carcinoma (HCC) remains unclear. Multiple databases including The Cancer Genome Atlas, Gene Expression Omnibus and Kaplan-Meier plotter were used for bioinformatics analysis of the $P P A R \gamma$ gene or protein. Immunohistochemical labeling of tumor and adjacent normal tissues obtained from 125 patients with $\mathrm{HCC}$ was performed to analyze the relationship between PPAR $\gamma$ expression and overall survival (OS) rate. PPAR $\gamma$ was evaluated using functional enrichment analyses and Lasso regression was used to conduct a dimensionality reduction analysis of 43 clinical factors for HCC. An OS prognostic nomogram was then established using seven independent risk factors screened via Lasso regression. PPAR $\gamma$ expression in HCC tumor tissues was higher compared with that in normal liver tissues, and its high expression was
\end{abstract}

Correspondence to: Dr Yemeng Wang, Department of Hepatobiliary Surgery, Zhuji People's Hospital of Zhejiang Province, 9 Jianmin Road, Zhuji, Zhejiang 311800, P.R. China

E-mail: doctorwym@aliyun.com

Dr Wensheng Pan, Department of Gastroenterology, Zhejiang Provincial People's Hospital, People's Hospital of Hangzhou Medical College, 158 Shangtang Road, Hangzhou, Zhejiang 310014, P.R. China

E-mail: wspan223@163.com

Key words: hepatocellular carcinoma, peroxisome proliferatoractivated receptor $\gamma$, risk factors, overall survival prognostic nomogram associated with poor prognosis, as indicated by bioinformatics analysis. However, opposite results were obtained using the clinical specimens. Functional enrichment analysis indicated that PPAR $\gamma$ was enriched in the 'fatty acid metabolism' pathway. Lasso regression identified seven clinical factors associated with prognosis, including Tumor-Node-Metastasis stage, grade, vascular invasion, $\alpha$ fetoprotein, carbohydrate antigen 199, $\gamma$-glutamyl transpeptidase and the PPAR $\gamma$ protein. These seven clinical factors were to construct an OS prognostic nomogram. Overall, PPAR $\gamma$ was highly expressed in the livers of patients with HCC and can be included in an OS prognostic nomogram. However, the factors underlying the differential association of PPAR $\gamma$ expression with HCC prognosis in different datasets should be further investigated.

\section{Introduction}

Hepatocellular carcinoma (HCC) is the most commonly occurring form of primary liver cancer and is one of the leading causes of cancer-associated death worldwide (1). HCC has poor prognosis and accounts for $75-85 \%$ of all primary liver cancer cases according to global cancer statistics in 2018 (2). Current therapies against HCC include ablation, liver transplantation and radical resection, which is the main treatment (3). However, HCC shows a high recurrence. Roayaie et al (4) reported that the recurrence rate of $\mathrm{HCC}$ is $60 \%$ at 5 years after surgery (4). Another study reported $\mathrm{HCC}$ recurrence, including true recurrence due to dissemination and de novo tumors within the oncogenic liver, makes $70 \%$ of cases worsen within 5 years after surgery (5). These have become the main problems that limit the 5-year survival rate of patients with liver cancer. This highlights the need to determine molecular mechanisms involved in the development of HCC and identify molecules that can be used in anti-HCC therapy. Developing new methods for predicting the prognosis of this disease is also important for 
improving the prognosis prediction of patients with HCC may help inform treatment choices.

Peroxisome proliferator activated receptor $\gamma(\operatorname{PPAR} \gamma)$, a ligand-activated transcription factor, is a member of the nuclear receptor superfamily of PPAR proteins. PPAR $\gamma$ has two subtypes: PPAR $\alpha$ And PPAR $\beta$ (6). PPAR $\alpha$ is involved in regulating various processes, from inflammation and immunity to nutrient metabolism and energy balance (7). PPAR $\beta$ has been shown to be involved in metabolism, angiogenesis and inflammatory responses (8). PPAR $\gamma$ plays an important role in the occurrence and development of various diseases, including obesity, inflammation, diabetes, atherosclerosis and cancer (9). This protein is also a major regulator of fat cell formation. In adipocytes, PPAR $\gamma$ regulates the expression of genes controlling the uptake and storage of free fatty acids and mediates the endocrine function of adipose tissue (10). A previous study showed that PPAR $\gamma$ may be involved in the occurrence and development of liver cancer (11). However, the specific mechanisms involved are unclear. It is also undetermined whether PPAR $\gamma$ acts as an antitumor or tumor-promoting factor in liver cancer (12).

Patients with HCC usually have a background of chronic liver disease such as non-alcoholic fatty liver disease (NAFLD), and chronic hepatitis B and C (13). NAFLD, a disease closely associated with metabolic syndrome, includes chronic liver diseases ranging from simple steatosis to non-alcoholic steatohepatitis (NASH) (14). Increasing epidemiological evidence indicates that NAFLD is a major cause of HCC (15). Numerous studies have shown that PPAR $\gamma$ is involved in the pathogenesis of NAFLD $(10,15-20)$. Elevated PPAR $\gamma$ levels can increase the expression of genes responsible for lipid metabolism in the liver, which induces liver steatosis and leads to NAFLD (21-23). Therefore, based on the previously identified role of PPAR $\gamma$ in liver cancer (24-26), the present study hypothesized that PPAR $\gamma$ may be involved in the development of HCC by affecting the metabolism of liver fat cells.

The present study examined the role of PPAR $\gamma$ in HCC development. Databases including The Cancer Genome Atlas (TCGA), Gene Expression Omnibus (GEO) and Kaplan-Meier plotter were used to predict the expression and function of PPAR $\gamma$ using bioinformatics. Then, the differences between the results obtained via bioinformatics analysis and those obtained by analysis of clinical data of 125 patients with HCC were compared. It was explored whether PPAR $\gamma$ participates in the development and progression of HCC by affecting lipid metabolism. Finally, PPAR $\gamma$ expression profiles and other selected prognostic clinical indicators were used to build a model for predicting the prognosis of patients with HCC.

\section{Materials and methods}

Differential analysis of PPARG $m R N A$ expression in patients with HCC conducted using TCGA and GEO databases. The data on PPAR $\gamma$ expression were downloaded from TCGA database (https://genome-cancer.ucsc.edu/) and GEO database (https://www.ncbi.nlm.nih.gov/geo/), then analyzed using the limma package of $\mathrm{R}$ software (version 3.5.2.) (27). The Wilcoxon signed-rank test was used to evaluate $P P A R G$ expression in HCC tissues and normal adjacent tissues of patients with HCC.
Interactive gene expression profiling. Gene Expression Profiling Interactive Analysis (GEPIA) (http://gepia. cancer-pku.cn/) is an interactive web application based on 9,736 tumors and 8,587 samples of normal tissue from TCGA and Genotype-Tissue Expression databases. GEPIA can be used for the profiling of cancerous and normal gene expression, and for interactive analyses (28). This database was used to explore the relationship between PPAR $\gamma$ expression levels and prognosis in patients with HCC.

Kaplan-Meier plotter analysis. The Kaplan-Meier plotter (http://kmplot.com/analysis/) database contains gene expression and clinical data for 21 different types of cancer including liver, lung, ovarian, gastric and breast cancer (29). Kaplan-Meier plotter was used to evaluate the prognostic value of PPAR in patients with HCC. The median PPAR expression (score of 1; Table I) was used as the cut-off value, which was used to divide patients into high and low expression groups and the prognosis of the two groups was compared.

Analysis of the human protein atlas. The Human Protein Atlas (https://www.proteinatlas.org), an open-access resource, was used to map all the human proteins in cells, tissues and organs. This mapping was performed to explore the distribution of PPAR $\gamma$ protein in tumor and adjacent normal tissues of patients with HCC.

Analysis of kyoto encyclopedia of genes and genomes (KEGG) and gene set enrichment analysis (GSEA). GSEA (http://www.broadinstitute.org/gsea/index.jsp) is a powerful analytical method that can interpret genome-wide expression profiles (30). KEGG enrichment analysis (31) for PPAR $\gamma$ was performed using The Database for Annotation, Visualization and Integrated Discovery (https://david.ncifcrf.gov), and evaluated the PPAR $\gamma$ pathways using GSEA. These analyses were performed using the clusterProfiler package of $\mathrm{R}$ software (version 3.5.2 (32).

Clinical factors affecting HCC development and progression. The study was approved by The Ethics Committee of the Zhejiang Provincial People's Hospital (Hangzhou, China). Clinical data on 125 patients with HCC were collected from the Zhejiang Provincial People's Hospital. The diagnosis of HCC in all the patients included was confirmed using histopathological examination by independent pathologists. Paraffin samples of HCC were collected in March 2020. These patients underwent surgical operations at Zhejiang Provincial People's Hospital from January 2008 to December 2015, and patients provided written informed consent at the time of tissue collection. The patients had complete follow-up data from the day of surgical resection of the primary tumor to death or the last follow-up. The last follow-up date was March 2016. The Tumor-Node-Metastasis (TNM) stage was defined according to the criteria described in the 8th American Joint Committee on Cancer guidelines (33). The inclusion criteria were as follows: i) Patients who underwent surgery, ii) patients with the histological type hepatocellular carcinoma and iii) patients with complete follow-up information. The exclusion criteria included: i) patients with other malignant diseases and (II) those without detailed clinical information. In addition, 43 clinical factors, including PPAR $\gamma$ protein 
Table I. Specific scoring criteria for staining intensity and proportion of stained cells.

Points

\begin{tabular}{|c|c|c|c|c|c|}
\hline \multirow[b]{2}{*}{ Specific criteria } & \\
\hline & 0 & 1 & 2 & 3 & 4 \\
\hline Dyeing intensity & Absent & Weak positive & Moderately positive & Strong positive & Strong positive \\
\hline Percentage range of positively stained cells, $\%$ & 0 & $1-25$ & $26-50$ & $51-75$ & $76-100$ \\
\hline
\end{tabular}

expression, were collected for all the patients (Table SI). Lasso regression was then performed on these clinical factors using the glmnet package (version 4.0-2.) in R software (http://www. jstatsoft.org/v33/i01/) (34). Univariate Cox regression analysis was also performed using $\mathrm{R}$ software on the selected clinical factors. Finally, the selected factors were used to establish a nomogram model using the rms, foreign and survival package in R software. The area under the curve (AUC) of receiver operating characteristic (ROC) and concordance index (C-index) were used to evaluate the accuracy of the model. Meanwhile, in order to validate that PPAR $\gamma$ did play a role in the established nomogram, other clinical factors besides PPAR $\gamma$ were used to establish another nomogram to obtain its $\mathrm{C}$-index and AUC for comparison.

Tissue microarray and immunohistochemical (IHC) analyses. Specimens that included tumor tissue and corresponding adjacent normal tissues were collected from 125 patients with HCC. A tissue microarray was constructed using paired tumor tissue and adjacent normal tissue collected $0.5-1.0 \mathrm{~cm}$ from the margin of the tumor (35). Immunolabeling of the tissue microarray was performed using an anti-PPAR $\gamma$ polyclonal antibody (1:200; cat. no. ab209350; Abcam). Briefly, sections from the tissue microarray were baked at $70^{\circ} \mathrm{C}$ for $2 \mathrm{~h}$ and then deparaffinized using xylene (cat. no. 534596; Sigma-Aldrich; Merck KGaA). A gradient of ethanol concentrations (100, 95 and $80 \%$ ) was used to rehydrate the sections, and antigen retrieval was performed by boiling the sections using a high-pressure cooker for $3 \mathrm{~min}$ in $1 \mathrm{mM}$ Tris-EDTA buffer. The sections were blocked with $3 \%$ hydrogen peroxide at room temperature for $15 \mathrm{~min}$ to inhibit endogenous peroxidase activity. Sections were then incubated with $10 \%$ goat non-immune serum (cat. no. ZLI-9022; Zsbio Store) for $20 \mathrm{~min}$ to reduce non-specific staining. Consequently, the sections were incubated with the primary antibody at $4^{\circ} \mathrm{C}$ overnight, and then with the Biotin-SP-AffiniPure Goat Anti-Rabbit IgG (H+L) (cat. no. 111-065-144; 1:500; Jackson ImmunoResearch Europe, Ltd.) at room temperature for $15 \mathrm{~min}$. Afterwards, the sections were incubated with HRP-conjugated streptavidin (cat. no. 3999s; CST Biological Reagents Co., Ltd.) at room temperature for another $15 \mathrm{~min}$. Protein expression was visualized using a diaminobenzidine substrate kit (cat. no. ab64238; 1:50; Abcam) and hematoxylin was used for $5 \mathrm{~min}$ at room temperature to counterstain the sections.

Evaluation of IHC labeling. Two experienced pathologists, blinded to the patients' pathology reports, independently scored the results of IHC labeling based on intensity and proportion of positively stained cells by light microscopy (cat. no. NI-SS 933679; Nikon Corporation). Signal intensity was expressed as: 0 for absent, 1 for weak positive, 2 for moderately positive and 3 for strong positive. The proportion of positively stained cells was also quantified as: 0 for $0 \%$ positively stained cells, 1 for $1-25 \%$ positively stained cells, 2 for $26-50 \%$ positively stained cells, 3 for $51-75 \%$ positively stained cells and 4 for $76-100 \%$ positively stained cells. Specific evaluation criteria are shown in Table I. The final score, obtained by multiplying the intensity score by the percentage of positively labeled cells, was used to indicate the expression of the PPAR $\gamma$ protein. A score of $\leq 1$ was defined as low PPAR $\gamma$ expression, while that of $>1$ was defined as high PPAR $\gamma$ expression.

Statistical analysis. Kaplan-Meier analysis was performed using log-rank tests. Lasso regression was used to assess the prognosis of patients with HCC and identify potential risk factors, and Lasso analysis sampled and analyzed the sample 1,000 times. Univariate Cox regression analysis was used to analyze the prognosis of clinical factors screened by Lasso analysis. The $\chi^{2}$ test was used to examine the association between PPAR $\gamma$ and other clinical factors in categorical variables. When variables with $\geq 20 \%$ of the cells had a count of $\leq 5$, the data were analyzed using Fisher's exact test. Additionally, the ROC curve was plotted and the AUC was calculated. The $\mathrm{C}$-index and AUC were used to analyze the accuracy of the nomogram. All statistical analyses were performed using the $\mathrm{R}$ software package. Confidence interval (CI) was set to $95 \%$. $\mathrm{P}<0.05$ was considered to indicate a statistically significant difference.

\section{Results}

Bioinformatics analysis of PPAR $\gamma$ mRA and protein expression. The results of the bioinformatics analysis showed that PPAR $\gamma$ mRNA expression in HCC tumor tissues was higher compared with that in normal tissues obtained from TCGA database $\left(\mathrm{P}=6.561 \times 10^{-11}\right.$; Fig. $\left.1 \mathrm{~A}\right)$. High PPARG expression was indicative of poor prognosis in patients with $\mathrm{HCC}$ as assessed using GEPIA ( $\mathrm{P}=0.00074$; Fig. 1B). It was concluded that high $P P A R \gamma$ expression was detrimental to patient prognosis by using Kaplan-Meier plotter analysis $(\mathrm{P}=0.0014$; Fig. 1C). Overexpression of PPAR $\gamma$ protein in HCC tissues was predictive of unfavorable prognosis in patients with $\mathrm{HCC}$ as assessed using analysis of the Human Protein Atlas $(\mathrm{P}<0.001$; Fig. 1D). In summary, bioinformatics analysis reported that high PPAR $\gamma$ mRNA or protein level in tumor tissues compared with normal tissues indicated a poor prognosis. 
A



$\mathrm{C}$

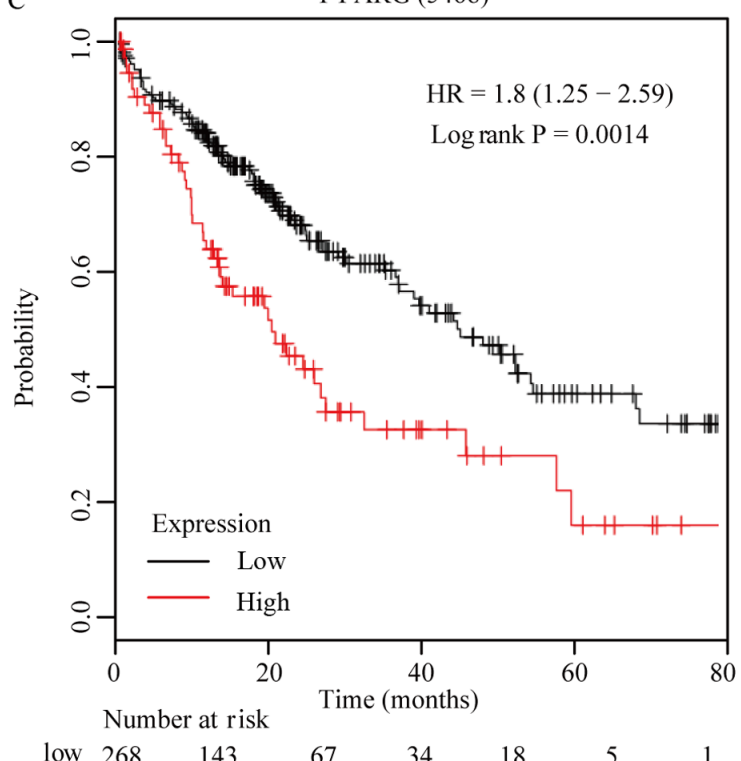

B



$\mathrm{D}$

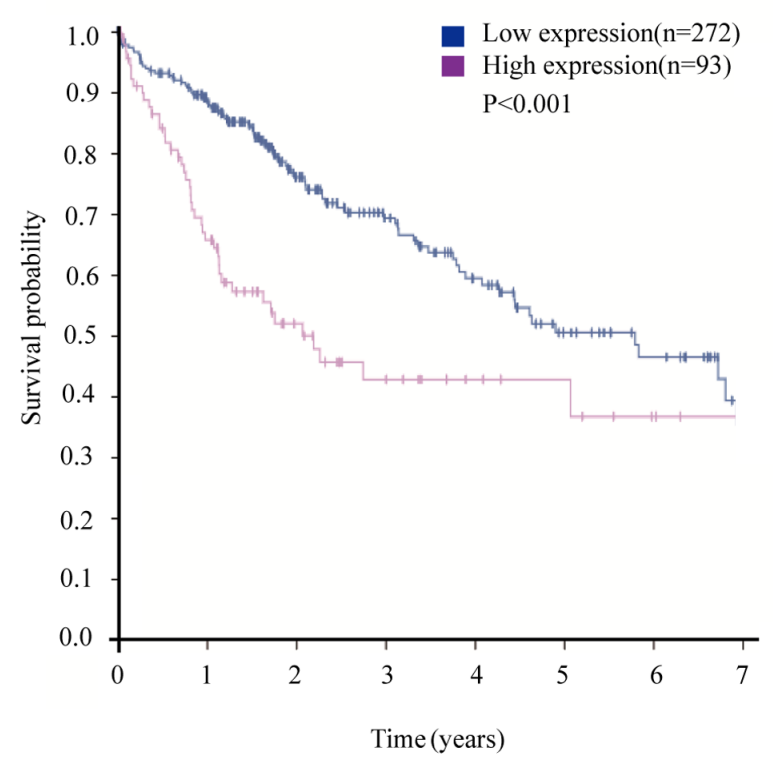

Figure 1. Bioinformatics analysis of PPAR $\gamma$ mRNA and protein expression. (A) mRNA expression of PPAR $\gamma$ in HCC tumor tissues was higher compared with that in adjacent normal tissues as assessed using The Cancer Genome Atlas database. (B) High PPAR $\gamma$ expression was associated with poor prognosis in patients with HCC as assessed using Gene Expression Profiling Interactive Analysis. (C) High PPAR $\gamma$ expression was associated with poor prognosis in patients with HCC as assessed using Kaplan-Meier plotter. (D) High expression of PPAR $\gamma$ protein in HCC tissues was unfavorable for prognosis in patients with HCC as assessed using analysis of the Human Protein Atlas. PPAR $\gamma$, peroxisome proliferator-activated receptor $\gamma$; HCC, hepatocellular carcinoma; HR, hazard ratio.

Relationship between PPAR $\gamma$ expression in HCC tissues and prognosis of patients with HCC. PPAR $\gamma$ expression in tumor tissues was higher compared with that in normal liver tissues by analysis of PPAR $\gamma$ expression in 125 patients with HCC ( $\mathrm{P}<0.001$; Fig. 2A), and the high expression of PPAR $\gamma$ was beneficial to the prognosis of patients $(\mathrm{P}=0.015$; Fig. 2B). IHC was used to evaluate PPAR $\gamma$ expression in tumor tissues and corresponding adjacent normal tissues. PPAR $\gamma$ expression in tissues was graded according to the criteria aforementioned.

Evaluation of IHC labeling. As shown in Fig. 3, PPAR $\gamma$ levels in tumor tissues (Fig. 3A and B) were higher compared with those in the adjacent normal tissues (Fig. 3C and D) of patients with HCC $(\mathrm{P}<0.001$; Fig. 3A). Increased expression of PPAR $\gamma$ in $\mathrm{HCC}$ tumor tissues was indicative of an improved prognosis in patients with $\mathrm{HCC}(\mathrm{P}=0.015$; Fig. 2B).

Association between PPAR $\gamma$ expression and clinical features in patients with HCC. The associations between PPAR $\gamma$ expression levels and key clinical characteristics of patients with HCC were examined (Table II). The results indicated that three clinical features including age $(\mathrm{P}=0.019)$, tumor diameter $(\mathrm{P}=0.041)$ and vascular invasion $(\mathrm{P}=0.026)$ were significantly associated with PPAR $\gamma$ expression. However, PPAR $\gamma$ expression was not significantly associated with factors such as hepatitis B, cirrhosis and metabolism-related indicators (Table II).

Functional enrichment analyses for PPAR $\gamma$. KEGG pathway enrichment was conducted through GSEA in high-expression 
A

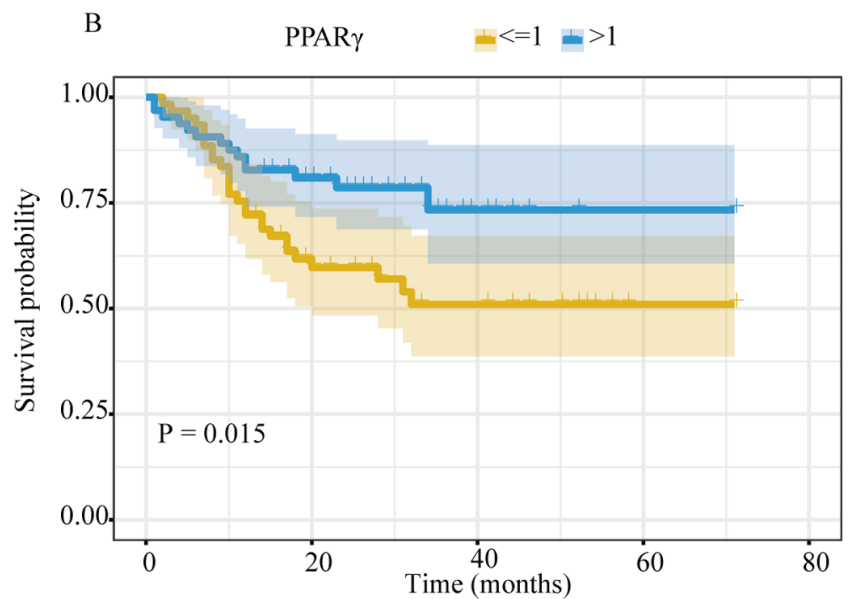

Figure 2. Relationship between PPAR $\gamma$ expression in HCC tissues and prognosis of patients with HCC. (A) PPAR $\gamma$ expression level in tumor tissues was higher compared with those in adjacent normal tissues of 125 patients with hepatocellular carcinoma. (B) High expression of PPAR $\gamma$ was associated with improved prognosis. PPAR $\gamma$, peroxisome proliferator-activated receptor $\gamma ; \mathrm{HCC}$, hepatocellular carcinoma.

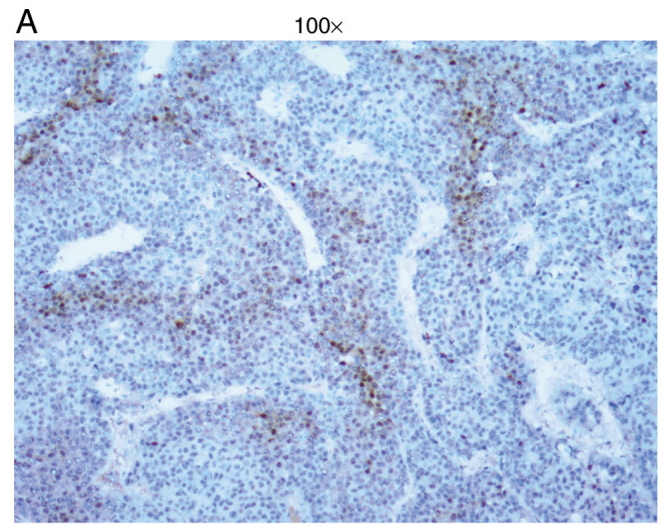

C

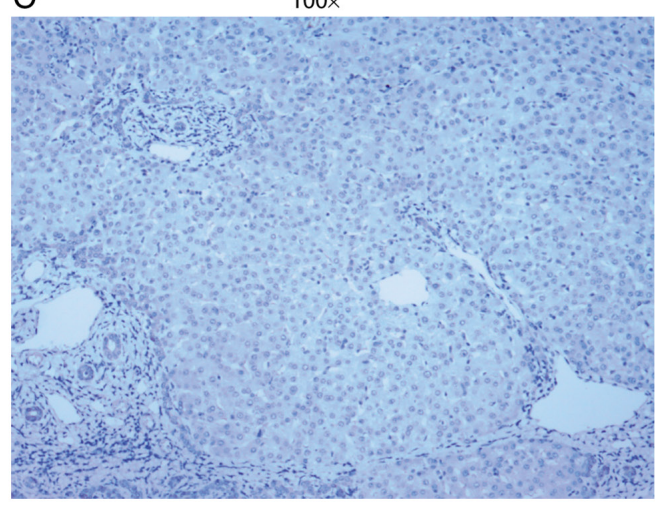

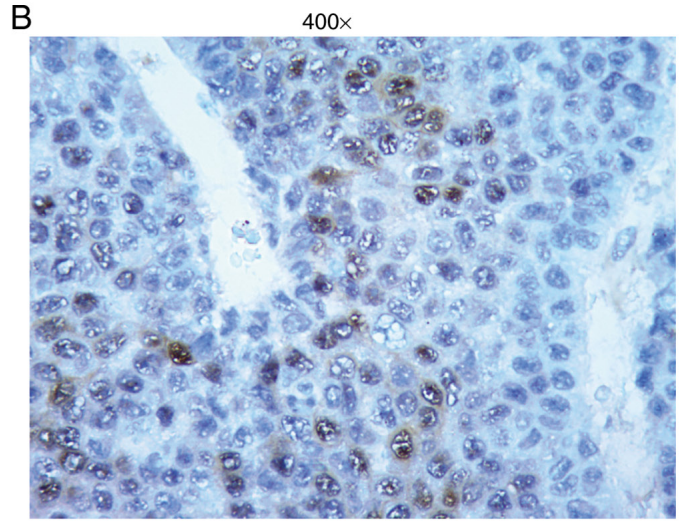

$\mathrm{D}$

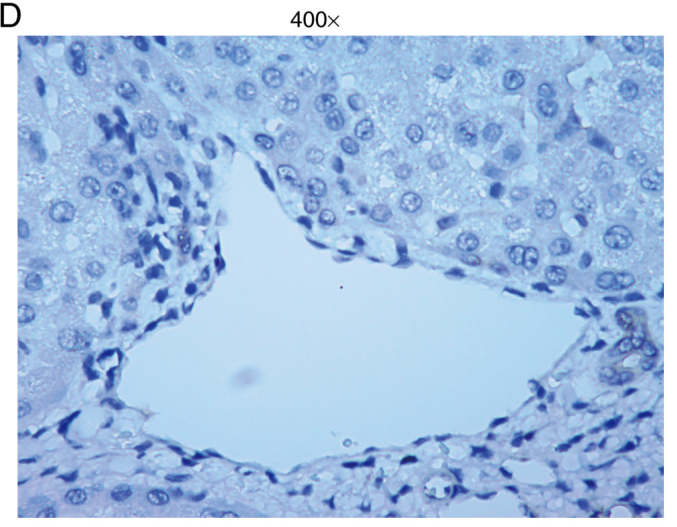

Figure 3. Peroxisome proliferator-activated receptor $\gamma$ expression in tumor tissues at (A) 100x and (B) 400x magnification and in the adjacent normal tissues at (C) 100x and (D) 400x magnification of patients with hepatocellular carcinoma.

group in the entire set by $\mathrm{R}$ software (clusterProfiler package). It revealed that patients with low PPAR $\gamma$ expression were associated with some pathways, including 'mitotic spindle,' ' $\mathrm{G}_{2} \mathrm{M}$ checkpoint,' 'E2F targets', 'spermatogenesis', 'mammalian target of rapamycin complex 1 signaling', 'fatty acid metabolism', 'TNFA signaling via NFKB' and 'xenobiotic metabolism' (Fig. 4A and B). Adjusted $\mathrm{P}<0.05$ for a gene set were considered statistically significant. Then a GSEA pathway analysis was performed with respect to fatty acid metabolism pathways (Fig. 4C). This indicated that the high expression of PPAR $\gamma$ gene in liver cancer is closely related to fatty acid metabolism.

Screening for clinical factors associated with prognosis. Lasso regression was used to analyze the 43 clinical factors aforementioned (Table SI), and identified the following seven clinical factors associated with prognosis: Tumor-Node-Metastasis (TNM) stage, type of differentiation, vascular invasion, $\alpha$ fetoprotein (APF), carbohydrate antigen 199 (CA199), $\gamma$-glutamyl transpeptidase ( $\gamma$-GT) and PPAR $\gamma$ protein expression (Fig. 5). 
Table II. Association between PPAR $\gamma$ and the clinical characteristics.

PPAR $\gamma$ expression

\begin{tabular}{|c|c|c|c|c|c|}
\hline & & & & & \\
\hline Factors & Low & High & Total, n & $\chi^{2}$ & P-value \\
\hline Sex & & & & 0.629 & 0.428 \\
\hline Male & 46 & 52 & 98 & & \\
\hline Female & 15 & 12 & 27 & & \\
\hline Age, years & & & & 5.464 & 0.019 \\
\hline$\leq 60$ & 43 & 32 & 75 & & \\
\hline$>60$ & 18 & 32 & 50 & & \\
\hline Tumor diameter, $\mathrm{cm}$ & & & & 4.163 & 0.041 \\
\hline$\leq 4$ & 29 & 42 & 71 & & \\
\hline$>4$ & 32 & 22 & 54 & & \\
\hline Grade & & & & 2.132 & 0.144 \\
\hline $\mathrm{I}+\mathrm{II}$ & 45 & 54 & 99 & & \\
\hline III & 16 & 10 & 26 & & \\
\hline TNM stage & & & & 0.642 & 0.663 \\
\hline $\mathrm{IA}+\mathrm{IB}+\mathrm{II}$ & 56 & 61 & 117 & & \\
\hline IIIA+IIIB+IVA+IVB & 5 & 3 & 8 & & \\
\hline $\mathrm{AFP}, \mu \mathrm{g} / 1$ & & & & 0.321 & 0.571 \\
\hline$\leq 200$ & 41 & 46 & 87 & & \\
\hline$>200$ & 20 & 18 & 38 & & \\
\hline Vascular invasion & & & & 4.979 & 0.026 \\
\hline Negative & 31 & 45 & 76 & & \\
\hline Positive & 30 & 19 & 49 & & \\
\hline Hepatitis B & & & & 0.261 & 0.609 \\
\hline Negative & 12 & 15 & 27 & & \\
\hline Positive & 49 & 49 & 98 & & \\
\hline Cirrhosis & & & & 0.913 & 0.339 \\
\hline Negative & 21 & 17 & 38 & & \\
\hline Positive & 40 & 47 & 87 & & \\
\hline $\mathrm{TG}, \mathrm{mmol} / \mathrm{l}$ & & & & 2.243 & 0.134 \\
\hline$\leq 1$ & 31 & 41 & 72 & & \\
\hline$>1$ & 30 & 23 & 53 & & \\
\hline $\mathrm{HDL}, \mathrm{mmol} / \mathrm{l}$ & & & & 0.001 & 0.990 \\
\hline$\leq 1.04$ & 42 & 44 & 86 & & \\
\hline$>1.04$ & 19 & 20 & 39 & & \\
\hline $\mathrm{LDL}, \mathrm{mmol} / \mathrm{l}$ & & & & 0.003 & 0.954 \\
\hline$\leq 2.1$ & 34 & 36 & 70 & & \\
\hline$>2.1$ & 27 & 28 & 55 & & \\
\hline TCHO, mmol/1 & & & & 0.473 & 0.492 \\
\hline$\leq 3.11$ & 23 & 28 & 51 & & \\
\hline$>3.11$ & 38 & 36 & 74 & & \\
\hline
\end{tabular}

AFP, $\alpha$ fetoprotein; TCHO, total cholesterol; TG, triglyceride; LDL, low density lipoprotein; HDL, high density lipoprotein; TNM, Tumor-Node-Metastasis.

Univariate analysis of the seven clinical factors associated with prognosis. Univariate analysis was performed on the seven clinical factors of TNM stage, grade, vascular invasion, APF, CA199, $\gamma$-GT and PPAR $\gamma$ protein expression that were screened previously using Lasso regression analysis. The results indicated that TNM stage ( $\mathrm{P}=0.005$; Table III), grade $(\mathrm{P}<0.001$; Table III $)$, vascular invasion $(\mathrm{P}<0.001$; Table III), APF ( $\mathrm{P}=0.035$; Table III), CA199 ( $\mathrm{P}=0.024$; 

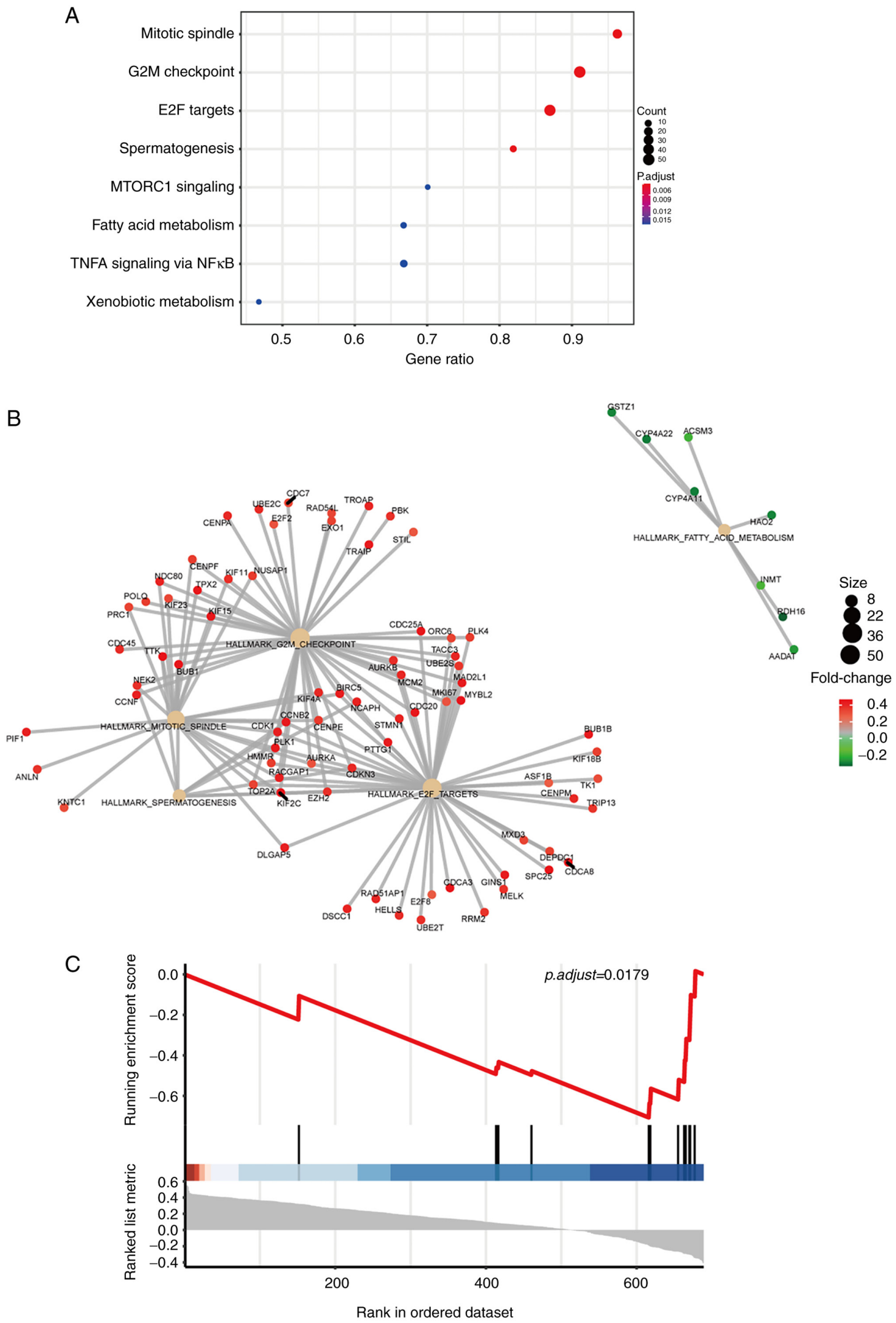

Figure 4. Functional enrichment analyses for PPAR $\gamma$. (A) Pathway enrichment of PPAR $\gamma$. (B) Genes associated with $P P A R \gamma$-enriched pathways. (C) Gene Set Enrichment Analysis of fatty acid metabolism pathways. PPAR $\gamma$, peroxisome proliferator-activated receptor $\gamma$; MTORC1, mammalian target of rapamycin complex 1 .

Table III), $\gamma$-GT ( $\mathrm{P}=0.002$; Table III) and expression of PPAR $\gamma$ protein $(\mathrm{P}=0.015$; Table III) were statistically significant factors affecting survival of patients with HCC
(Table III). These results indicated that that these seven factors were independent risk factors for the prognosis of patients with HCC. 


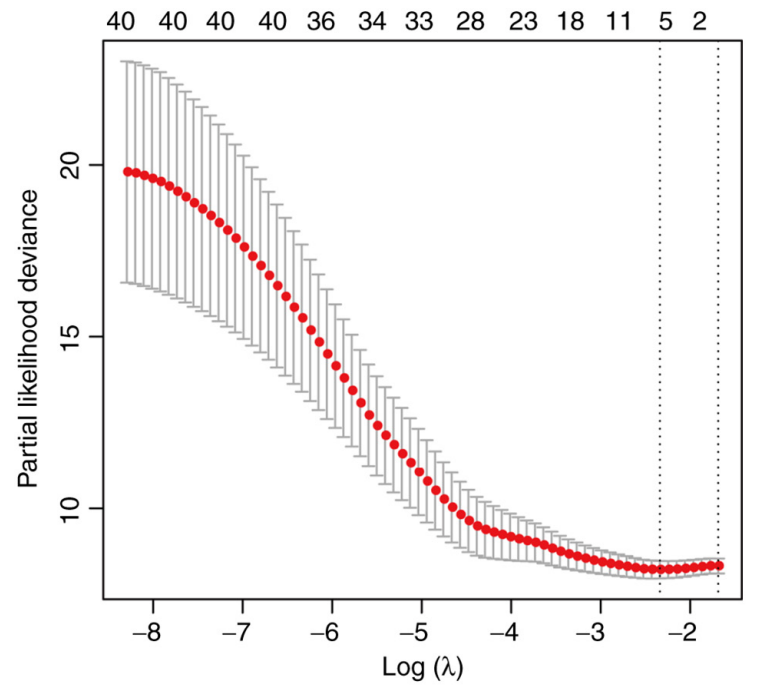

Figure 5. Lasso regression analysis of 43 clinical factors was used to screen out seven factors associated with the prognosis of patients with hepatocellular carcinoma. Seven clinical factors included: Tumor-Node-Metastasis stage, type of differentiation, vascular invasion, $\alpha$ fetoprotein, carbohydrate antigen 199, $\gamma$-glutamyl transpeptidase and peroxisome proliferator-activated receptor $\gamma$ protein expression.

Construction of an OS prognostic nomogram. The aforementioned seven clinical factors were used to build an effective OS prognostic nomogram to predict the prognosis of patients with HCC (Fig. 6). The C-index used to evaluate the accuracy of the nomogram using these clinical factors was 0.755 (95\% CI, 0.591 to 0.919 ; $\mathrm{P}<0.001$; data not shown) and the AUC values predicting the 1-, 3- and 5-year OS of the nomogram were $0.744,0.780$ and 0.780 , respectively (Fig. 7A-C). As for the nomogram established by clinical factors that did not include PPAR $\gamma$, the C-index and AUC values were both lower. The C-index was 0.748 (95\% CI, 0.584 to $0.912 ; \mathrm{P}<0.001$; data not shown). The AUC values predicting the 1-, 3- and 5-year OS of the nomogram were 0.706, 0.751 and 0.751 , respectively (Fig. 7D-F).

\section{Discussion}

$\mathrm{HCC}$ is a major human health concern because of its high rates of recurrence and metastasis (36). Elucidating the molecular regulatory mechanisms involved in $\mathrm{HCC}$ is necessary for improving diagnostic methods and anti-HCC therapies. This requires detailed understanding of the molecular regulatory mechanisms involved in HCC (37). PPAR $\gamma$ is potentially involved in mediation of HCC-related mechanisms $(38,39)$. However, the role of PPAR $\gamma$ expression in HCC remains controversial. Some studies have shown high expression of PPAR $\gamma$ protein in HCC tissues (40-42), while another report indicated that PPAR $\gamma$ protein expression is decreased in HCC (43). Presently, PPAR $\gamma$ is considered to exert an antitumor effect in HCC because PPAR $\gamma$ has been identified as a tumor suppressor gene (44). PPAR $\gamma$ plays a key role in the proliferation, migration, invasion and apoptosis of HCC cells. Cao et al (45) have demonstrated that PPAR $\gamma$ activation can inhibit the proliferation of liver hepatic cancer cells by downregulating septin 2 expression. Lee et al (46) reported

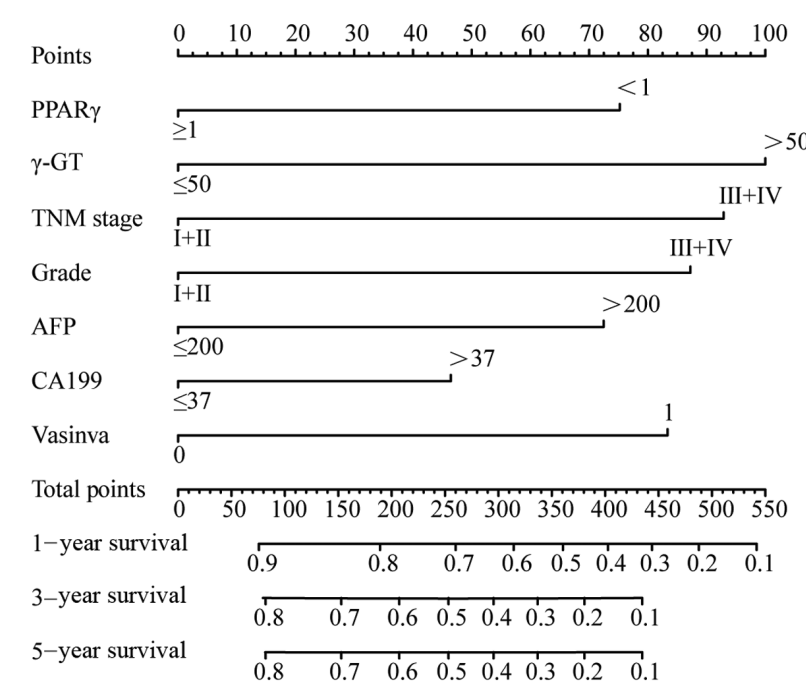

Figure 6. In total, seven clinical factors, including TNM stage, grade, vascular invasion, APF, CA199, $\gamma$-GT and PPAR $\gamma$ protein expression, were used to establish an overall survival prognostic nomogram. PPAR $\gamma$, peroxisome proliferator-activated receptor $\gamma$; TNM, Tumor-Node-Metastasis; AFP, $\alpha$ fetoprotein; CA199, carbohydrate antigen 199; $\gamma$-GT, $\gamma$-glutamyl transpeptidase.

that PPAR $\gamma$ may be involved in modulating E-cadherin expression and motility of HepG2 cells. Another study demonstrated that PPAR $\gamma$ can directly inhibit the migration of HCC cells and is negatively correlated with macrovascular invasion observed in HCC (47). Han et al (48) showed that hispidulin inhibits the growth and metastasis of HCC via PPAR $\gamma$ activation, mediated by $A M P K$ and $E R K$ signaling. In summary, previous studies indicate that high expression of PPAR $\gamma$ in liver cancer exerts a robust tumor-suppressive effect.

The present study first employed bioinformatics analyses to establish that PPAR $\gamma$ expression in tumor tissues of patients with HCC was higher compared with that in adjacent normal liver tissues. PPAR $\gamma$ expression was examined in 125 clinical samples collected from patients with $\mathrm{HCC}$ by performing tissue microarray and IHC analyses and concluded that PPAR $\gamma$ was highly expressed in liver tumor tissues. These results showed that PPAR $\gamma$ can be used as a tumor biomarker in HCC.

The relationship between PPAR $\gamma$ expression levels and several key clinical characteristics in patients with HCC were also evaluated. The results indicated that PPAR $\gamma$ expression is associated with age, tumor diameter and vascular invasion. However, PPAR $\gamma$ expression was not significantly associated with factors such as hepatitis B, cirrhosis and metabolism-related indicators. These results concurred with the results of a previous study by Hsu et al (47).

Bioinformatics analyses using multiple databases was used to evaluate the relationship between PPAR $\gamma$ expression and prognosis of patients with HCC. The results showed that increased PPAR $\gamma$ protein or gene expression was indicative of a less favorable prognosis, which contradicted the results from previous studies $(11,36,44)$. To explain this discrepancy, the relationship between expression levels of PPAR $\gamma$ and prognosis of 125 patients with HCC was evaluated. The results indicated that high expression of PPAR $\gamma$ was indicative of improved prognosis. The inconsistency between these results 
Table III. Univariate analysis of survival in 125 patients with hepatocellular carcinoma.

\begin{tabular}{|c|c|c|c|c|}
\hline Factors & Value, $\mathrm{n}$ & $\mathrm{P}$-value & HR & P-value (HR) \\
\hline \multicolumn{5}{|l|}{$\operatorname{PPAR} \gamma$} \\
\hline$\leq 1$ & 61 & 0.015 & Reference & 0.018 \\
\hline$>1$ & 64 & & $0.459(0.240-0.875)$ & \\
\hline \multicolumn{5}{|l|}{ Grade } \\
\hline $\mathrm{I}+\mathrm{II}$ & 99 & $<0.001$ & Reference & 0.001 \\
\hline III & 26 & & $2.848(1.519-5.341)$ & \\
\hline \multicolumn{5}{|l|}{ TNM stage } \\
\hline $\mathrm{IA}+\mathrm{IB}+\mathrm{II}$ & 117 & 0.005 & Reference & 0.009 \\
\hline IIIA+IIIB+IVA+IVB & 8 & & $3.537(1.372-9.116)$ & \\
\hline \multicolumn{5}{|l|}{ Vascular invasion } \\
\hline Negative & 76 & $<0.001$ & Reference & 0.001 \\
\hline Positive & 49 & & $2.748(1.475-5.121)$ & \\
\hline \multicolumn{5}{|l|}{$\mathrm{AFP}, \mu \mathrm{g} / \mathrm{l}$} \\
\hline$\leq 200$ & 87 & 0.035 & Reference & 0.039 \\
\hline$>200$ & 38 & & $1.928(1.035-3.593)$ & \\
\hline \multicolumn{5}{|l|}{$\mathrm{CA} 199, \mu \mathrm{g} / \mathrm{ml}$} \\
\hline$\leq 37$ & 65 & 0.024 & Reference & 0.027 \\
\hline$>37$ & 60 & & $2.141(1.09-4.025)$ & \\
\hline \multicolumn{5}{|l|}{$\gamma-\mathrm{GT}, \mathrm{U} / 1$} \\
\hline$\leq 51$ & 66 & 0.002 & Reference & 0.003 \\
\hline$>51$ & 59 & & 2.707 (1.417-5.17) & \\
\hline
\end{tabular}

PPAR $\gamma$, peroxisome proliferator activated receptor $\gamma$;AFP, $\alpha$ fetoprotein; $\gamma$-GT, $\gamma$-glutamyl transpeptidase; CA199, carbohydrate antigen 199; TNM, Tumor-Node-Metastasis; HR, hazard ratio.

A 1-year survival AUC $=0.744$

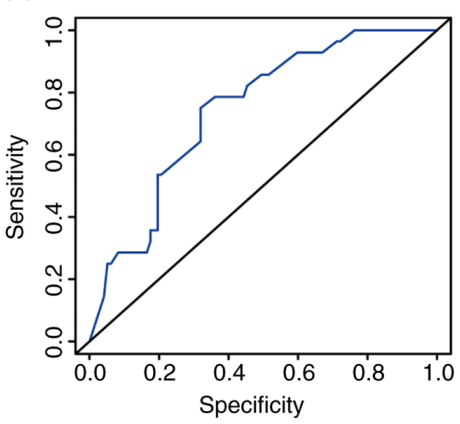

D 1-year survival $\mathrm{AUC}=0.706$

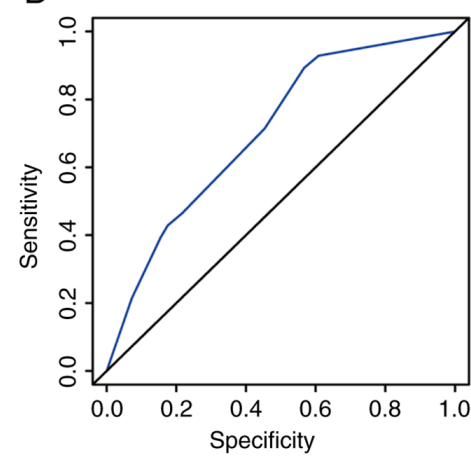

B 3-year survival AUC $=0.78$

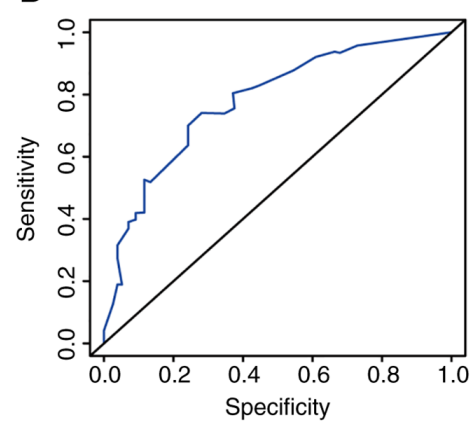

E 3-year survival $A U C=0.751$

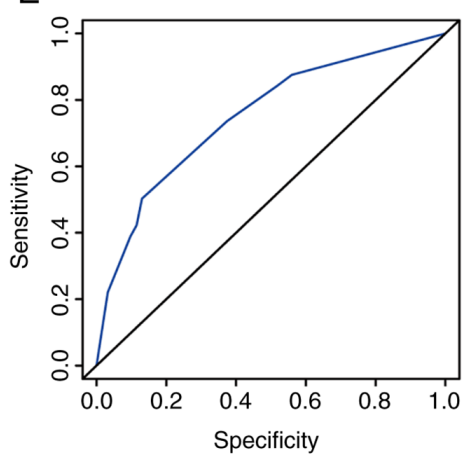

C 5-year survival $\mathrm{AUC}=0.78$



F 5-year survival $A U C=0.751$

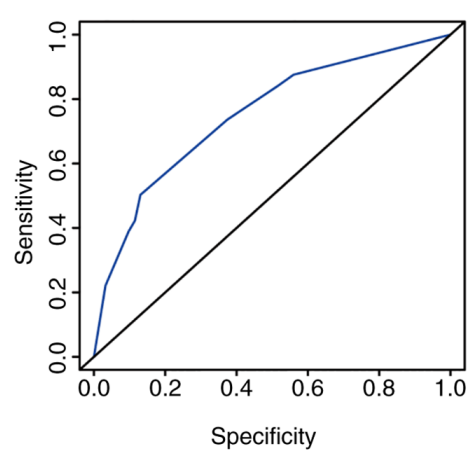

Figure 7. AUC values predict the 1-, 3- and 5-year OS of the nomogram that used seven clinical factors including PPAR $\gamma$. ROC curve prediction for (A) 1-, (B) 3- and (C) 5-year OS. AUC values predicted the 1-, 3- and 5-year OS of the nomogram that used six clinical factors without PPAR . ROC curve for prediction of (D) 1- (E) 3- and (F) 5-year OS. AUC, area under the curve; ROC, receiver operating characteristic; PPAR $\gamma$, peroxisome proliferator-activated receptor $\gamma$. 
and those obtained using bioinformatics analysis may be due to the following reasons. The data used for the bioinformatics analyses was collected on patients in Western countries, while the present patient cohort included patients residing in China, which may leads to bias in prognostic results. Second, China has a high incidence of hepatitis B, and most cases of liver cancer in China are caused by hepatitis B (49). However, in Western countries, most liver cancer cases are caused by over-consumption of alcohol (50). Therefore, the different pathogenesis of liver cancer may account for the differences in prognosis. Third, due to differences in eating habits, Westerners weigh more than East Asians (51). PPAR $\gamma$ expression may be affected by differences in body weights. These differences in eating habits and weight likely contribute to differences in prognosis. In addition, as the two sets of data to be analyzed have different sources, other factors (such as ethnicity, weight and treatment method) for the two sets of data cannot be controlled. At the same time, there is research showing that PPAR $\gamma$ mRNA and protein levels do not have the same expression level, which indicates that there may be post-transcriptional modifications in HCC (11).

Although the present study did not observe an association between PPAR $\gamma$ expression and related indices of lipid metabolism, it was reported that PPAR $\gamma$ was indeed enriched in the 'fatty acid metabolism' pathway. Changes in liver metabolism are critical to the development of liver disease. PPAR $\gamma$ is involved in mitochondrial oxidative phosphorylation, gluconeogenesis and fatty acid synthesis. Since modifications that affect mitochondria and lipid metabolism all contribute to the occurrence and/or development of liver steatosis, NAFLD, NASH and HCC, the function of PPAR $\gamma$ in lipid metabolism is closely related to the occurrence and development of liver cancer (25). This observation will be investigated further in our future study into the role of PPAR $\gamma$ in liver cancer pathogenesis.

To make the present study more comprehensive and accurate, data on a total of 43 clinical factors for patients with $\mathrm{HCC}$ were collected. First, Lasso regression was used to perform a dimensionality reduction analysis on these factors. Consequently, seven indicators were identified, including PPAR $\gamma$ expression, that were closely associated with the prognosis of patients with HCC. A univariate analysis was then performed on these indicators to verify the results obtained using Lasso analysis. The results of univariate analysis confirmed that these seven factors were indeed independent risk factors associated with the prognosis of patients with HCC. Finally, these prognosis-associated factors were used to establish an OS prognostic nomogram for rapidly and accurately predicting HCC prognosis. The C-index and AUC values of the nomogram established with PPAR $\gamma$ were higher compared with those of the nomogram established without PPAR $\gamma$, which further demonstrated that the expression of PPAR $\gamma$ may play a role in predicting the prognosis of patients with HCC.

The present study used several innovative approaches. First, PPAR $\gamma$ protein and gene expression was assessed using bioinformatics analyses. Then Lasso analysis was used to identify clinical and comprehensive factors associated with patient prognosis. These data demonstrated that PPAR $\gamma$ was associated with prognosis in patients with HCC. As single prognostic factors play limited roles in prognosis (52), a OS prognostic nomogram based on independent factors, such as PPAR $\gamma$ expression, was constructed to predict the OS rate in patients with HCC. This type of nomogram, which has been used previously as a prognostic prediction model, integrates all prognostic factors to achieve an individualized prediction (53). Additionally, this nomogram incorporates an enhanced visual interface and is straightforward to operate, which is advantageous in clinical practice (54). The limitations of the present study were as follows. First, semi-quantitative analysis was used to evaluate the results of PPAR $\gamma$ immunolabeling assay, which may have led to statistical bias. Second, the insufficient number of samples collected could not rule out the possibility of sampling error. Additionally, single-center data were used to build the prognostic model and was not verified using external data. Lastly, the role of PPAR $\gamma$ in HCC pathogenesis needs to be explored in greater detail. PPAR $\gamma$ should be knocked down or overexpressed in cells to observe its effect on tumor progression and specific mechanisms in in vivo and in vitro experiments, which will be the subject of our future studies.

The present study reported that PPAR $\gamma$ was highly expressed in the tumor tissues of patients with $\mathrm{HCC}$, and its expression level was associated with age, tumor diameter and vascular invasion. These results indicated that PPAR $\gamma$ expression can be used as a biomarker for predicting the prognosis of HCC. The OS prognostic nomogram, established using clinical factors and PPAR $\gamma$ expression levels, can be used to rapidly and accurately predict the prognosis of patients with HCC, leading to improved monitoring of the present patient population.

\section{Acknowledgements}

Not applicable.

\section{Funding}

The study was funded by The Program on the Funds of Science Technology Department of Zhejiang province (grant. no. LGF19H160027) and The General Project Funds from the Health Department of Zhejiang Province (grant. no. 2018259783).

\section{Availability of data and materials}

The datasets used and/or analyzed during the present study are available from the corresponding author on reasonable request. The additional datasets analyzed during the current study are available in The Cancer Genome Atlas database (https:/genome-cancer.ucsc.edu/), Gene Expression Omnibus database (https://www.ncbi.nlm.nih.gov/geo/) and The Human Protein Atlas (https://www.proteinatlas.org).

\section{Authors' contributions}

XLZ designed the study. WP provided administrative support and made substantial contributions to the conception. XZ collected the data. ZD and TT performed data analysis. YC contributed to manuscript revisions and participated in data 
collection and sorting. YW was involved in revising the manuscript critically for important intellectual content, and analysis and interpretation of data. XLZ, WP, YC and YW confirm the authenticity of all raw data. All authors read and approved the final manuscript.

\section{Ethics approval and consent to participate}

The study was approved by The Ethics Committee of the Zhejiang Provincial People's Hospital (Hangzhou, China; approval no. 2020QT103).

\section{Patient consent for publication}

The study received an informed consent exemption approved by The Ethics Committee of the Zhejiang Provincial People's Hospital.

\section{Competing interests}

The authors declare that they have no competing interests.

\section{References}

1. Baffy G, Brunt EM and Caldwell SH: Hepatocellular carcinoma in non-alcoholic fatty liver disease: an emerging menace. J Hepatol 56: 1384-1391, 2012.

2. Bray F, Ferlay J, Soerjomataram I, Siegel RL, Torre LA and Jemal A: Global cancer statistics 2018: GLOBOCAN estimates of incidence and mortality worldwide for 36 cancers in 185 countries. CA Cancer J Clin 68: 394-424, 2018.

3. El-Serag HB, Marrero JA, Rudolph L and Reddy KR: Diagnosis and treatment of hepatocellular carcinoma. Gastroenterology 134 1752-1763, 2008

4. Roayaie S, Obeidat K, Sposito C, Mariani L, Bhoori S, Pellegrinelli A, Labow D, Llovet JM, Schwartz M and Mazzaferro V: Resection of hepatocellular cancer $\leq 2 \mathrm{~cm}$ : Results from two Western centers. Hepatology 57: 1426-1435, 2013.

5. Forner A, Reig M and Bruix J: Hepatocellular carcinoma. Lancet 391: 1301-1314, 2018.

6. Yousefnia S, Momenzadeh S, Seyed Forootan F, Ghaedi K and Esfahani MH: The influence of peroxisome proliferator-activated receptor $\gamma$ (PPAR $\gamma)$ ligands on cancer cell tumorigenicity. Gene 649: 14-22, 2018.

7. Li S, Yang B, Du Y, Lin Y, Liu J, Huang S, Zhang A, Jia Z and Zhang Y: Targeting PPAR $\alpha$ for the treatment and understanding of cardiovascular diseases. Cell Physiol Biochem 51: 2760-2775, 2018.

8. Magadum A and Engel FB: PPAR $\beta / \delta$ : Linking metabolism to regeneration. Int J Mol Sci 19: 2013, 2018.

9. Lehrke M and Lazar MA: The many faces of PPARgamma. Cell 123: 993-999, 2005.

10. Skat-Rørdam J, Højland Ipsen D, Lykkesfeldt J and Tveden-Nyborg P: A role of peroxisome proliferator-activated receptor $\gamma$ in non-alcoholic fatty liver disease. Basic Clin Pharmacol Toxicol 124: 528-537, 2019.

11. Hsu HT and Chi CW: Emerging role of the peroxisome proliferator-activated receptor-gamma in hepatocellular carcinoma. J Hepatocell Carcinoma 1: 127-135, 2014.

12. Lee YH, Seo D, Choi KJ, Andersen JB, Won MA, Kitade M, Gómez-Quiroz LE, Judge AD, Marquardt JU, Raggi C, et al: Antitumor effects in hepatocarcinoma of isoform-selective inhibition of HDAC2. Cancer Res 74: 4752-4761, 2014.

13. Giannini EG, Aglitti A, Borzio M, Gambato M, Guarino M, Iavarone M, Lai Q, Levi Sandri GB, Melandro F, Morisco F, et al: Overview of immune checkpoint inhibitors therapy for hepatocellular carcinoma, and The ITA.LI.CA cohort derived estimate of amenability rate to immune checkpoint inhibitors in clinical practice. Cancers (Basel) 11: 1689, 2019.

14. Anstee QM, Reeves HL, Kotsiliti E, Govaere O and Heikenwalder M: From NASH to HCC: Current concepts and future challenges. Nat Rev Gastroenterol Hepatol 16: 411-428, 2019.
15. Choudhary NS, Kumar N and Duseja A: Peroxisome proliferator-activated receptors and their agonists in nonalcoholic fatty liver disease. J Clin Exp Hepatol 9: 731-739, 2019.

16. Westerbacka J, Kolak M, Kiviluoto T, Arkkila P, Sirén J, Hamsten A, Fisher RM and Yki-Järvinen H: Genes involved in fatty acid partitioning and binding, lipolysis, monocyte/macrophage recruitment, and inflammation are overexpressed in the human fatty liver of insulin-resistant subjects. Diabetes 56: 2759-2765, 2007.

17. Tanaka N, Aoyama T, Kimura S and Gonzalez FJ: Targeting nuclear receptors for the treatment of fatty liver disease. Pharmacol Ther 179: 142-157, 2017.

18. Feng X, Yu W, Li X, Zhou F, Zhang W, Shen Q, Li J, Zhang C and Shen P: Apigenin, a modulator of PPAR $\gamma$, attenuates HFD-induced NAFLD by regulating hepatocyte lipid metabolism and oxidative stress via Nrf2 activation. Biochem Pharmacol 136: 136-149, 2017.

19. Samuel VT and Shulman GI: Nonalcoholic fatty liver disease as a nexus of metabolic and hepatic diseases. Cell Metab 27: 22-41, 2018

20. Silva AKS and Peixoto CA: Role of peroxisome proliferatoractivated receptors in non-alcoholic fatty liver disease inflammation. Cell Mol Life Sci 75: 2951-2961, 2018.

21. Gao M, Ma Y, Alsaggar M and Liu D: Dual outcomes of rosiglitazone treatment on fatty liver. AAPS J 18: 1023-1031, 2016.

22. Yang SJ, Choi JM, Chae SW, Kim WJ, Park SE, Rhee EJ, Lee WY, Oh KW, Park SW, Kim SW and Park CY: Activation of peroxisome proliferator-activated receptor gamma by rosiglitazone increases sirt6 expression and ameliorates hepatic steatosis in rats. PLoS One 6: e17057, 2011.

23. Yu S, Matsusue K, Kashireddy P, Cao WQ, Yeldandi V, Yeldandi AV, Rao MS, Gonzalez FJ and Reddy JK: Adipocyte-specific gene expression and adipogenic steatosis in the mouse liver due to peroxisome proliferator-activated receptor gamma1 (PPARgamma1) overexpression. J Biol Chem 278: 498-505, 2003

24. Feng J, Dai W, Mao Y, Wu L, Li J, Chen K, Yu Q, Kong R, Li S, Zhang J, et al: Simvastatin re-sensitizes hepatocellular carcinoma cells to sorafenib by inhibiting HIF-1 $\alpha /$ PPAR- $\gamma /$ PKM2-mediated glycolysis. J Exp Clin Cancer Res 39: 24, 2020.

25. Piccinin E, Villani G and Moschetta A: Metabolic aspects in NAFLD, NASH and hepatocellular carcinoma: The role of PGC1 coactivators. Nat Rev Gastroenterol Hepatol 16: 160-174, 2019.

26. Shu Y, Lu Y, Pang X, Zheng W, Huang Y, Li J, Ji J, Zhang C and Shen P: Phosphorylation of PPAR $\gamma$ at Ser84 promotes glycolysis and cell proliferation in hepatocellular carcinoma by targeting PFKFB4. Oncotarget 7: 76984-76994, 2016.

27. Ritchie ME, Phipson B, Wu D, Hu Y,Law CW, Shi W and Smyth GK: limma powers differential expression analyses for RNA-sequencing and microarray studies. Nucleic Acids Res 43: e47, 2015.

28. Tang Z, Li C, Kang B, Gao G, Li C and Zhang Z: GEPIA: A web server for cancer and normal gene expression profiling and interactive analyses. Nucleic Acids Res 45 (W1): W98-W102, 2017.

29. Hou GX, Liu P, Yang J and Wen S: Mining expression and prognosis of topoisomerase isoforms in non-small-cell lung cancer by using Oncomine and Kaplan-Meier plotter. PLoS One 12: e 0174515,2017

30. Subramanian A, Tamayo P, Mootha VK, Mukherjee S, Ebert BL, Gillette MA, Paulovich A, Pomeroy SL, Golub TR, Lander ES and Mesirov JP: Gene set enrichment analysis: A knowledge-based approach for interpreting genome-wide expression profiles. Proc Natl Acad Sci USA 102: 15545-15550, 2005.

31. Ogata H, Goto S, Sato K, Fujibuchi W, Bono H and Kanehisa M: KEGG: Kyoto encyclopedia of genes and genomes. Nucleic acids Res 27: 29-34, 1999.

32. R Core Team: R: A language and environment for statistical computing. R Foundation for Statistical Computing, Vienna, Austria. ISBN 3-900051-07-0, 2012. URL: http://www.R-project.org/.

33. Bierley JD, Gospodarowicz MK and Wittekind C (eds): The TNM classification of malignant tumours. 8th edition. Oxford, Wiley, Blackwell, 2017.

34. Friedman J, Hastie T and Tibshirani R: Regularization paths for generalized linear models via coordinate descent. J Stat Softw 33: 1-22, 2010.

35. Chen S, Dong Z, Yang P, Wang X, Jin G, Yu H, Chen L, Li L, Tang L, Bai S, et al: Hepatitis B virus X protein stimulates high mobility group box 1 secretion and enhances hepatocellular carcinoma metastasis. Cancer Lett 394: 22-32, 2017.

36. Nojima H, Kuboki S, Shinoda K, Shimizu H, Ohtsuka M, Kato A Yoshitomi H, Furukawa K, Takayashiki T and Miyazaki M: Activation of peroxisome proliferator-activated receptor-gamma inhibits tumor growth by negatively regulating nuclear factor- $\kappa \mathrm{B}$ activation in patients with hepatocellular carcinoma. J Hepatobiliary Pancreat Sci 23: 574-584, 2016. 
37. Cervello M,Emma MR,Augello G,Cusimano A,GiannitrapaniL, Soresi M, Akula SM, Abrams SL, Steelman LS, Gulino A, et al: New landscapes and horizons in hepatocellular carcinoma therapy. Aging (Albany NY) 12: 3053-3094, 2020.

38. Xu Z, Meng SH, Bai JG, Sun C, Zhao LL, Tang RF, Yin ZL, Ji JW, Yang W and Ma GJ: C/EBP $\alpha$ regulates FOXC1 to modulate tumor growth by interacting with PPAR $\gamma$ in hepatocellular carcinoma. Curr Cancer Drug Targets 20: 59-66, 2020.

39. Tan J, Shen W, Shi W, Chen X, Sun D, Xu C, Yan Q, Cheng H, Lai Y and Ji H: ONTD induces growth arrest and apoptosis of human hepatoma Bel-7402 cells though a peroxisome proliferator-activated receptor $\gamma$-dependent pathway. Toxicol In Vitro 45: 44-53, 2017.

40. Schaefer KL, Wada K, Takahashi H, Matsuhashi N, Ohnishi S, Wolfe MM, Turner JR, Nakajima A, Borkan SC and Saubermann LJ: Peroxisome proliferator-activated receptor gamma inhibition prevents adhesion to the extracellular matrix and induces anoikis in hepatocellular carcinoma cells. Cancer Res 65: 2251-2259, 2005.

41. Yu J, Shen B, Chu ES, Teoh N, Cheung KF, Wu CW, Wang S, Lam CN, Feng H, Zhao J, et al: Inhibitory role of peroxisome proliferator-activated receptor gamma in hepatocarcinogenesis in mice and in vitro. Hepatology 51: 2008-2019, 2010.

42. Koga H, Sakisaka S, Harada M, Takagi T, Hanada S, Taniguchi E, Kawaguchi T, Sasatomi K, Kimura R, Hashimoto O, et al: Involvement of p21(WAF1/Cip1), p27(Kip1), and p18(INK4c) in troglitazone-induced cell-cycle arrest in human hepatoma cel lines. Hepatology 33: 1087-1097, 2001.

43. Yu J, Qiao L, Zimmermann L, Ebert MP, Zhang H, Lin W, Röcken C, Malfertheiner P and Farrell GC: Troglitazone inhibits tumor growth in hepatocellular carcinoma in vitro and in vivo. Hepatology 43: 134-143, 2006.

44. Wu CW, Farrell GC and Yu J: Functional role of peroxisome-proliferator-activated receptor gamma in hepatocellular carcinoma. J Gastroenterol Hepatol 27: 1665-1669, 2012.

45. Cao LQ, Shao ZL, Liang HH, Zhang DW, Yang XW, Jiang XF and Xue P: Activation of peroxisome proliferator-activated receptor- $\gamma(\mathrm{PPAR} \gamma)$ inhibits hepatoma cell growth via downregulation of SEPT2 expression. Cancer Lett 359: 127-135, 2015.
46. Lee HJ, Su Y, Yin PH, Lee $\mathrm{HC}$ and $\mathrm{Chi} \mathrm{CW}$ : PPAR(gamma)/PGC-1(alpha) pathway in E-cadherin expression and motility of HepG2 cells. Anticancer Res 29: 5057-5063, 2009.

47. Hsu HT, Sung MT, Lee CC, Kuo YJ, Chi CW, Lee HC and Hsia CY: Peroxisome proliferator-activated receptor $\gamma$ expression is inversely associated with macroscopic vascular invasion in human hepatocellular carcinoma. Int J Mol Sci 17: 1226, 2016.

48. Han M, Gao H, Ju P, Gao MQ, Yuan YP, Chen XH, Liu KL, Han YT and Han ZW: Hispidulin inhibits hepatocellular carcinoma growth and metastasis through AMPK and ERK signaling mediated activation of PPAR $\gamma$. Biomed Pharmacother 103: 272-283, 2018

49. Lin L, Yan L, Liu Y, Qu C, Ni J and Li H: The burden and trends of primary liver cancer caused by specific etiologies from 1990 to 2017 at the global, regional, national, age, and sex level results from the global burden of disease study 2017. Liver Cancer 9: 563-582, 2020.

50. Singal AG, Lampertico P and Nahon P: Epidemiology and surveillance for hepatocellular carcinoma: New trends. J Hepatol 72: 250-261, 2020.

51. Oliveros E, Somers VK, Sochor O, Goel K and Lopez-Jimenez F: The concept of normal weight obesity. Prog Cardiovasc Dis 56: 426-433, 2014.

52. Tu Q, Hu C, Zhang H, Peng C, Kong M, Song M, Zhao C, Wang Y, Li J,Zhou C, et al: Establishment and validation of novel clinical prognosis nomograms for luminal a breast cancer patients with bone metastasis. Biomed Res Int 2020: 1972064, 2020.

53. Zhang X, Yuan K, Wang H, Gong P, Jiang T, Xie Y, Sheng L, Liu D, Liu X and Xu G: Nomogram to predict mortality of endovascular thrombectomy for ischemic stroke despite successful recanalization. J Am Heart Assoc 9: e014899, 2020.

54. Bookman MA: Can we predict who lives long with ovarian cancer? Cancer 125 (Suppl 24): S4578-S4581, 2019.

This work is licensed under a Creative Commons Attribution-NonCommercial-NoDerivatives 4.0 International (CC BY-NC-ND 4.0) License. 